\title{
VERTEX ALGEBRA GENERATION OF ALMOST HOLOMORPHIC MODULAR FORMS
}

\author{
ALEXANDER ZUEVSKY \\ Institute of Mathematics \\ Academy of Sciences of the Czech Republic \\ Prague \\ e-mail: zuevsky@yahoo.com
}

\begin{abstract}
Using computations related to vertex operator algebra characters, we derive the simplest example of almost holomorphic modular forms.
\end{abstract}

\section{Introduction}

It is well-known $[9,12,14]$ that vertex operator algebras $[2,6,9]$ serve as a natural algebraic source of automorphic forms generation. In this short note, we show how to generate the simplest example of almost holomorphic modular form [3] $\hat{E}_{2}(\tau)=E_{2}(\tau)+c / y$, where $E_{2}(\tau)$ is the second Eisenstein series [13], $y=\operatorname{Im}(\tau), \tau$ is the torus modular parameter, and $c$ is a constant. For this purpose, we use the machinery of character computations developed for vertex operator algebras/conformal field theory [12]. The holomorphic quasi-modular form $E_{2}(\tau)$ appeared at various points in computations related to vertex operator algebra module. At the same time, it was not know how to generate its non-holomorphic

2010 Mathematics Subject Classification: 11F03, 30F10, 17B69, 32A25.

Keywords and phrases: automorphic forms, non-holomorphic extension of Eisenstein series, vertex algebras.

Received January 16, 2015

(ㄷ) 2015 Scientific Advances Publishers 
extension $\hat{E}_{2}(\tau)$ using similar reasoning. The author is also grateful to Bringmann for pointing the problem and useful discussions. In this note, we find answer for this question.

\subsection{Almost holomorphic modular forms}

Almost holmorphic modular forms [3], which originally defined in [8], transform like usual modular forms, but are polynomials in $1 / v$ with holomorphic coefficients. Standard examples of almost holomorphic modular form include derivatives of holomorphic modular forms, as well as the non-holomorphic Eisenstein series $\hat{E}_{2}(\tau)$, defined by

$$
\hat{E}_{2}(\tau)=E_{2}(\tau)+\frac{c}{\operatorname{Im} \tau}
$$

where $c=-3 / \pi$ Here its holomorphic part is given by

$$
E_{2}(\tau)=1-24 \sum_{n \geq 1} \sigma_{1}(n) q^{n}
$$

where $\sigma_{1}(n)$ is the sum of positive integer divisors of $n$, and $q=e^{2 \pi i \tau}$. In general, the holomorphic part of an almost holomorphic modular form is called a quasimodular form. After their introduction [8], almost holomorphic modular forms have been shown to play numerous roles in mathematics and physics (see, for example, [1]).

\subsection{Vertex operator algebras}

Recall that a vertex operator algebra [2, 6, 9] is a quadruple $(V, Y, \mathbf{1}, \omega)$ consisting of a $\mathbb{Z}$-graded complex vector space $V=\bigoplus_{n \in \mathbb{Z}} V_{n}$, a linear map $Y: V \rightarrow(\operatorname{End} V)\left[\left[z, z^{-1}\right]\right]$, and a pair of distinguished vectors, i.e., the vacuum vector $1 \in V_{0}$, and the conformal vector $\omega \in V_{2}$. A vertex operator is given by the map

$$
Y(v, z)=\sum_{n \in \mathbb{Z}} v(n) z^{-n-1},
$$

with modes $v(n) \in \operatorname{End} V$, where $\left.Y(v, z) \cdot \mathbf{1}\right|_{z=0}=v(-1) \cdot \mathbf{1}=v$, and $z$ is a formal variable. For the vector $\omega$, one has 


$$
Y(\omega, z)=\sum_{n \in \mathbb{Z}} L(n) z^{-n-2} .
$$

The modes $L(n)$ form the Virasoro Lie algebra of central charge $C$ :

$$
[L(m), L(n)]=(m-n) L(m+n)+\left(m^{3}-m\right) \delta_{m,-n} C / 12 .
$$

We define the homogeneous space of weight $k$ to be $V_{k}=\{v \in V \mid L(0) v=k v\}$, where for $v$ in $V_{k}$ we write $w t(v)=k$. Then as an operator on $V$, we have

$$
v(n): V_{m} \rightarrow V_{m+k-n-1} .
$$

In particular, the zero mode $o(v)=v(w t(v)-1)$ is a linear operator on each homogeneous space of $V$.

\subsection{The Heisenberg vertex operator algebra $M$}

Consider $a \in V_{1}$ whose modes obey

$$
[a(m), a(n)]=m \delta_{m,-n} I_{V}
$$

where $I_{V}$ is the identity operator on $V$. The vertex operator $Y(a, z)$ generates the Heisenberg vertex operator algebra $M$ with vector space $V$ with Fock basis

$$
a(-1)^{r_{1}} a(-2)^{r_{2}} \cdots a(-n)^{r_{n}} . \mathbf{1} ; \quad r_{i} \geq 0
$$

with $a=a(-1) .1$, and $a(n) .1=0$ for all $n \geq 0$. The Virasoro vector is $\omega=\frac{1}{2} a^{2}(-1) .1$ for central charge $C=1$ with

$$
L(0)=\frac{1}{2} a(0)^{2}+\sum_{m \geq 0} a(-m) a(m)
$$

In the context of modular-invariance for $n$-point functions at genus 1 , Zhu introduced in [14] a second vertex operator algebra $(V, Y[],, \mathbf{1}, \widetilde{\omega})$ associated to a given vertex operator algebra $(V, Y(),, \mathbf{1}, \omega)$. The 
underlying Fock space of the second vertex operator algebra is the same space $V$ as the first, moreover, they share the same vacuum vector $\mathbf{1}$ and have the same central charge. The new vertex operators are defined by a change of co-ordinates, namely,

$$
Y[v, z]=\sum_{n \in \mathbb{Z}} v[n] z^{-n-1}=Y\left(q_{z}^{L(0)} v, q_{z}-1\right)
$$

where $q_{z}=e^{z}$. The new conformal vector $\widetilde{\omega}$ is defined to be the state $\widetilde{\omega}=\omega-\frac{C}{24} .1$. We set

$$
Y[\widetilde{\omega}, z]=\sum_{n \in \mathbb{Z}} L[n] z^{-n-2},
$$

and write $w t[v]=k$ if $L[0] . v=k v, V_{[k]}=\{v \in V \mid w t[v]=k\}$. A Heisenberg vertex operator algebra module $M_{\alpha}$ is determined by

$$
a(n) w=\delta_{n, 0} \alpha \cdot w
$$

for all $w \in M_{\alpha}, n \geq 0$ for a complex $\alpha \in \mathbb{C}$.

\subsection{Torus two-point function}

Torus $n$-point function for a vertex operator algebra is defined by [14]

$$
F_{V}^{(1)}\left(v_{1}, z_{1} ; \ldots ; v_{n}, z_{n} ; q\right)=\operatorname{Tr}_{V}\left(Y\left(v_{1}, z_{1}\right) \ldots Y\left(v_{n}, z_{n}\right) q^{L(0)-C / 24}\right) .
$$

In particular, the partition (or zero-point) functions and one-point functions are given by

$$
\begin{gathered}
F_{V}^{(1)}(q)=\operatorname{Tr}_{V}\left(q^{L(0)-C / 24}\right) \\
F_{V}^{(1)}(v, z ; q)=\operatorname{Tr}_{V}\left(Y(v, z) q^{L(0)-C / 24}\right)=\operatorname{Tr}_{V}\left(o(v) q^{L(0)-C / 24}\right) .
\end{gathered}
$$

The second equality in the last formula follows from (1.1). Note that onepoint function does not depend on $z$. 
Modular and elliptic functions arise in context of computation of $n$-point functions for vertex operator algebras [12, 14]. The Zhu recursion formula [14] relates $n$-point functions to $(n-1)$-point functions. Thus, two-point function

$$
\begin{aligned}
F_{V}^{(1)}(u, x ; v, y ; q)= & \operatorname{Tr}_{V}\left(o(u) o(v) q^{L(0)-C / 24}\right) \\
& +\sum_{m \geq 0} \frac{1}{m !} \frac{\partial^{m}}{\partial y^{m}} P_{1}(x-y) F_{V}^{(1)}(u[m] v, q),
\end{aligned}
$$

for elliptic and modular functions

$$
P_{1}(z)=-\sum_{n \in \mathbb{Z}, n \neq 0} \frac{e^{n z}}{1-q^{n}}=\frac{1}{z}-\sum_{n \geq 1} E_{2 n}(q) z^{2 n-1}
$$

and

$$
E_{2 n}(\tau)=-\frac{B_{2 n}}{(2 n) !}+\frac{2}{(2 n-1) !} \sum_{r \geq 0} \frac{r^{2 n-1} q^{r}}{1-q^{r}}
$$

where $E_{2 n}(\tau)$ are Eisenstein series, and $B_{2 n}$ being Bernoulli numbers [13]. Note that $E_{2}(\tau)$ is a quasi-modular form satisfying the transformation law:

$$
(c \tau+d)^{-2} E_{2}(\gamma \tau)=E_{2}(\tau)-\frac{c}{2 \pi i(c \tau+d)}, \gamma=\left(\begin{array}{ll}
a & b \\
c & d
\end{array}\right), \gamma \tau=\frac{a \tau+b}{\gamma \tau+d},
$$

for $\gamma \in S L(2, \mathbb{Z})$.

\section{The Simplest Almost Holomorphic Modular Form}

\subsection{Identities for the Heisenberg vertex operator algebra}

$E_{2}(\tau)$ has also important relations with derivatives of modular forms. One can show that

$$
q \frac{d}{d q} \eta(\tau)=-\frac{1}{2} \eta(\tau) E_{2}(\tau)
$$


Note, in particular, that the one-point Virasoro vector function for the Heisenberg vertex operator algebra is given by [7]

$$
F_{M_{0}}^{(1)}(\widetilde{\omega})=q \frac{d}{d q} \eta(\tau)^{-1}=\frac{E_{2}(\tau)}{2 \eta(\tau)} .
$$

For the Virasoro vector $\widetilde{\omega}=\omega-\frac{C}{24}$, we find [12]

$$
\begin{aligned}
F_{V}^{(1)}(\widetilde{\omega}, q) & =\operatorname{Tr}_{V}\left((L(0)-C / 24) q^{L(0)-C / 24}\right)=q \partial_{q} F_{V}^{(1)}(q) \\
& =\frac{1}{2 \pi i} \partial_{\tau} F_{V}^{(1)}(q) .
\end{aligned}
$$

For $n$ primary (i.e., satisfying $L(n) u=0$, for all $n \geq 1$ ) vectors $u_{1}, \ldots, u_{n}$, the Zhu reduction gives the Ward identity [10, 12] for the $n+1$-point function:

$$
\begin{aligned}
& F_{V}^{(1)}\left(\tilde{\omega}, x ; u_{1}, z_{1} ; \ldots ; u_{n}, z_{n} ; q\right)=q \partial_{q} F_{V}^{(1)}\left(u_{1}, z_{1} ; \ldots ; u_{n}, z_{n} ; q\right) \\
& \quad+\sum_{1 \leq i \leq n}\left(P_{1}\left(x-z_{i}\right) \partial_{z_{i}}+w t\left[u_{i}\right] \partial_{z_{i}} P_{1}\left(x-z_{i}\right)\right) F_{V}^{(1)}\left(u_{1}, z_{1} ; \ldots ; u_{n}, z_{n} ; q\right) .
\end{aligned}
$$

$E_{2}(\tau)$ can be reproduced via the computation of a one-point function for the Heisenberg vertex operator algebra [11, 12, 14]. For the Heisenberg vertex operator algebra $M$, the Zhu reduction gives all $n$-pt functions, e.g., $[10,12]$

$$
F_{M}^{(1)}\left(a, z_{1} ; a ; z_{2} ; q\right)=P_{2}\left(z_{1}-z_{2}, \tau\right) F_{M}^{(1)}(q)
$$

where $P_{2}(z, \tau)=-\frac{d}{d z}, P_{1}(z, \tau)$. Then,

$$
\begin{aligned}
F_{M}^{(1)}(\widetilde{\omega}, q) & =\frac{1}{2} \lim _{x \rightarrow y}\left(F_{M}^{(1)}\left(a, z_{1} ; a, z_{2} ; q\right)-\left(z_{1}-z_{2}\right)^{-2} F_{M}^{(1)}(q)\right) \\
& =\frac{1}{2} E_{2}(q) F_{M}^{(1)}(q) .
\end{aligned}
$$


Thus one has an ordinary differential equation

$$
q \partial_{q} F_{M}^{(1)}(q)=\frac{1}{2} E_{2}(q) F_{M}^{(1)}(q)
$$

therefore, the genus one partition function for the Heisenberg vertex operator algebra is given by $F_{M}^{(1)}(q)=\frac{1}{\eta(q)}$.

\subsection{The simplest almost holomorphic modular form}

Since, as we saw in (2.1) that $E_{2}(\tau)$ is given by the Heisenberg vertex operator algebra one point function for the Virasoro vector $\widetilde{\omega},[12]$, we need to extend this vertex operator algebra in order to reconstruct the non-holomorphic part of $\hat{E}_{2}(\tau)$. For that purpose, we consider Virasoro state $\widetilde{\omega}$ one-point function for a Heisenberg module. We formulate the following:

Proposition 1. For $\alpha= \pm\left(\frac{c}{\operatorname{Im} \tau}\right)^{1 / 2}$, the torus Virasoro state character generates the simplest almost holomorphic modular form, i.e.,

$$
F_{M_{\alpha}}^{(1)}(\widetilde{\omega}, q)=\frac{1}{2} \hat{E}_{2}(\tau) F_{M_{\alpha}}^{(1)}(q)
$$

Proof. The Zhu reduction formula (1.2) also applies to $n$-pt correlation functions for a vertex operator algebra module [12]. For a Heisenberg module $M_{\alpha}$ (for some $\alpha \in \mathbb{C}$ ), one finds that

$$
F_{M_{\alpha}}^{(1)}(q)=\operatorname{Tr}_{M_{\alpha}}\left(q^{L}(0)-1 / 24\right)=\frac{q^{\alpha^{2}}}{\eta(q)}
$$

The one-point function for the Virasoro vector $\widetilde{\omega}$ is [7]

$$
F_{M_{\alpha}}^{(1)}(\widetilde{\omega}, q)=q \partial_{q} F_{M_{\alpha}}^{(1)}(q)
$$

On the other hand, the Zhu reduction formula (1.2) gives

$$
F_{M_{\alpha}}^{(1)}\left(a ; z_{1} ; a ; z_{2} ; q\right)=\left(\frac{\alpha^{2}}{2}+P_{2}\left(z_{1}-z_{2}\right)\right) F_{M_{\alpha}}^{(1)}(q)
$$


so that

$$
F_{M_{\alpha}}^{(1)}(\widetilde{\omega}, q)=\frac{1}{2}\left(\alpha^{2}+E_{2}(q)\right) F_{M_{\alpha}}^{(1)}(q)
$$

Now let us choose $\alpha= \pm\left(\frac{c}{\operatorname{Im} \tau}\right)^{1 / 2}$. Then we obtain

$$
F_{M_{\alpha}}^{(1)}(\widetilde{\omega}, q)=\frac{1}{2}\left(E_{2}(\tau)+\frac{c}{\operatorname{Im} \tau}\right) F_{M_{\alpha}}^{(1)}(q)=\frac{1}{2} \hat{E}_{2}(\tau) F_{M_{\alpha}}^{(1)}(q)
$$

Thus we see that the Zhu recursion formula (1.2) which is the main tool in computation of the torus as well as genus two (see [10]) $n$-point functions for vertex operator algebras results in generation of nonholomorphic modular forms as coefficients, and provides us with the simplest example of non-holomorphic quasi-modular forms. Similar reasoning can be used to derive [15] more complicated examples of mock forms $[3,16]$.

\section{References}

[1] M. Aganagic, V. Bouchard and A. Klemm, Topological strings and (almost) modular forms, Comm. Math. Phys. 277(3) (2008), 771-819.

[2] R. E. Borcherds, Vertex algebras, Kac-Moody algebras and the Monster, Proc. Nat. Acad. Sc. 83 (1986), 3068-3071.

[3] K. Bringmann and A. Folsom, Almost harmonic Maass forms and Kac-Wakimoto characters, To appear in J. Reine Angew. Math. (Crelle's Journal), arXiv:1112.4726, 2011.

[4] T. Creutzig and A. Milas, False Theta Functions and the Verlinde formula. arXiv: 1309.6037 (2013).

[5] T. Eguchi and Y. Sugawara, Non-holomorphic modular forms and $S L(2, \mathbb{R}) / U(1)$ superconformal field theory, JHEP, March 2011, 2011:107, arXiv:1012.5721.

[6] I. B. Frenkel, J. Lepowsky and A. Meurman, Vertex Operator Algebras and the Monster, Pure and Appl. Math., Vol. 134, Academic Press, Boston, 1988.

[7] D. Hurley and M. P. Tuite, Virasoro correlation functions for vertex operator algebras, Internat. J. Math. 23(10) (2012), 1250106. 
[8] M. Kaneko and D. Zaiger, A generalized Jacobi theta function and quasimodular forms, in: The moduli space of curves (Texel Island, 1994), 165-172, Progr. Math. 129, Birkhauser Boston, Massachusetts, 1995.

[9] V. Kac, Vertex Operator Algebras for Beginners, University Lecture Series 10, AMS, Providence, 1998.

[10] T. Gilroy and M. P. Tuite, To appear, 2014.

[11] G. Mason and M. P. Tuite, Torus chiral $n$-point functions for free boson and lattice vertex operator algebras, Comm. Math. Phys. 235(1) (2003), 47-68.

[12] G. Mason and M. P. Tuite, Vertex Operators and Modular Forms, A Window into Zeta and Modular Physics, 183-278, Math. Sci. Res. Inst. Publ., 57, Cambridge Univ. Press, Cambridge, 2010.

[13] J.-P. Serre, A Course in Arithmetic, Berlin: Springer-Verlag, 1978.

[14] Y. Zhu, Modular invariance of characters of vertex operator algebras, J. Amer. Math. Soc. 9 (1996), 237-302.

[15] A. Zuevsky, Mock forms from vertex algebras, To appear (2014).

[16] S. Zwegers, PhD Thesis, Mock Theta Functions, Utrecht University, 2002. 